\title{
Simulated Solar Microwave Radiation Blocks the Formation of Biofilms
}

\author{
Yulia S. Shishkova1, Stanislav N. Darovskih², Nadezhda V. Vdovina², \\ Nadezhda L. Pozdnyakova1, I. A. Komarova1, Elena V. Shishkova1, \\ Evgenij V. Vodyanitskiy ${ }^{2}$ \\ ${ }^{1}$ South Ural State Medical University, Chelyabinsk, Russia \\ ${ }^{2}$ South Ural State University, Chelyabinsk, Russia \\ Email: nadzh@yandex.ru
}

Received 16 February 2015; accepted 6 March 2015; published 10 March 2015

Copyright (C) 2015 by authors and Scientific Research Publishing Inc.

This work is licensed under the Creative Commons Attribution International License (CC BY). http://creativecommons.org/licenses/by/4.0/

c) (i) Open Access

\section{Abstract}

The article presents the results of the experimental study that was devoted to determining the blocking influence of the solar microwave radiation on the process of biofilm formation in Grampositive and Gram-negative microorganisms. The microwave generator that allows simulating microwave "splashes" of the Sun in the frequency range $(4.0-4.3 \mathrm{GHz})$ with the controlled intensity of radiation (from $50 \mu \mathrm{W} / \mathrm{sm}^{2}$ to $500 \mu \mathrm{W} / \mathrm{sm}^{2}$ ) was used for conducting this research. It is found out that the simulated solar radiation of the microwave range blocks the formation of the extracellular matrix by the opportunistic microorganisms. The results of this study confirm the hypothesis of the evolutionary nature of the leading role of the microwave radiation of the Sun in the life processes of organisms. The technology of the exposure on the microorganisms that was used in the experiment opens up the real prospects for reducing the persistent potential of microorganisms and improving the efficiency of the bacterial infections treatment.

\section{Keywords}

Solar Microwave Radiation, Microorganisms, Biofilms, Extracellular Matrix, Persistence

\section{Introduction}

The problem of the treatment of bacterial diseases becomes more and more relevant in modern clinical medicine. First, this is due to the progressive antibiotic resistance of microorganisms [1]. It has been known since 90s of $\mathrm{XX}$ century that the majority of bacteria do not exist as separate isolated cells, but as communities, which are immersed in the extracellular matrix, forming biofilm [2] [3]. Matrix structure, consisting mostly of polysaccha- 
rides, underlies the restricted access of antibiotics and other antimicrobial factors to microorganisms in biofilms [4]. As a part of a biofilm, bacteria are more resistant to immune system effectors, antibiotics and disinfectants. Nowadays the role of the biofilms in the development of chronic and/or recurrent infections has been determined [5].

Despite the large amount of the research works devoted to the problem of reducing the microbial resistance in biofilms, currently there are no constructive proposals on how to reduce the persistent potential of microorganisms.

All of the above is a reason for searching for the new approaches to development of the high-performance technologies aimed at weakening or blockading the formation of the extracellular matrix of microorganism in biofilms. Such technology must increase the availability of direct effect of the immune system's effectors and antimicrobial drugs on the persistent microorganisms, and may be the basis for prevention and treatment of a wide range of diseases of microbial etiology and the basis for reducing the number of complications, associated with them.

An important stage of the search for a way to reduce the persistent potential of microorganisms is to understand the main reasons of the amplification of the resistant properties of microorganisms. Many scientists believe that it may be related to the ecological aspects of the changes in the environment [6]. In recent decades, significant changes in the biosphere are associated with its electromagnetic pollution. At present time, the natural electromagnetic background is almost completely suppressed by anthropogenic radiation. In this connection, the leading role of the main source of microwave radiation in the nature, which is the Sun throughout the evolutionary development of organisms, becomes significantly less. And, according to the authors, this is the reason to enhance the process biofilm formation by microorganisms. Counteracting this process is necessary to restore the control role of natural electromagnetic factor. Achieving this objective in terms of electromagnetic pollution associates with the simulation of electromagnetic radiation amplified analog of natural origin in particular solar radiation microwave range [6]. The use of such radiation, according to the authors, will restore the modulatory role of microwave radiation on the formation of biofilms opportunistic microorganisms.

All of the above determines the main aim of our study: to evaluate the influence of the simulated solar radiation of microwave diapason on the process of biofilms formation.

\section{Materials and Methods}

To implement our idea the generator of microwave oscillations was created, which allows to simulate microwave "splashes" of the Sun in the frequency range (4.0 - 4.3) $\mathrm{GHz}$ with the controlled intensity of radiation (from $50 \mu \mathrm{W} / \mathrm{sm}^{2}$ to $500 \mu \mathrm{W} / \mathrm{sm}^{2}$ ) [7].

As an object for exposure by simulated microwave radiation of the Sun were selected daily cultures of $E$. coli, K. pneumonia, P. aeruginosa, S. aureus, which have the ability to form biofilms, isolated from the pathological material (urine, wound discharge, sputum, blood) from the patients with differently located inflammatory processes. Exposure time for these cultures was $16 \mathrm{~min}$. As a control group a part of the above-mentioned microorganism's cultures were left without any influence. Biofilm formation activity was measured after $24 \mathrm{~h}$ of cultivation by a photometric method, using magenta and the scanning electron microscope of high resolution JEOL JSM-6460 LV; the results were expressed in percentage [8]. This study was carried out in six replicates.

\section{Results}

As a result of the study we revealed that after the influence on a suspension of daily cultures of $E$. coli, $K$. pneumonia, $P$. aeruginosa, $S$. aureus of simulated solar microwave radiation in vitro, there was a significant decrease or complete loss of the ability to form biofilms by the tested microorganisms (Table 1).

For the visualization of these phenomena we performed scanning electron microscopy of the biofilms, which were formed during 24 hours by E. coli and S. aureus at the bottom of the plastic Petri plates without an influence (control) and after pre-exposure of microwave simulated solar radiation to the microorganisms suspension in a liquid growth medium.

The received microscopic picture of the control samples of Staphylococcus aureus (Figure 1) and Escherichia coli (Figure 2) fully responds to the contemporary conception of the biofilm organization of developed microorganisms' colony. The presented photos clearly show that the cells of Staphylococcus aureus (Figure 1) and Escherichia coli (Figure 2) are immersed into the extracellular matrix. Besides, in the biofilm formed by E. coli, the ejection of the single bacteria was observed, which reflects the logical dynamics of the processes, occurring 
Table 1. Biofilm formation of the tested microorganisms after an influence of simulated solar microwave radiation, $\%$.

\begin{tabular}{ccc}
\hline $\begin{array}{c}\text { The name of the } \\
\text { microorganism }\end{array}$ & Control group, \% & $\begin{array}{c}\text { After the influence, \% } \\
\mathrm{M} \pm \mathrm{m}\end{array}$ \\
\hline $\begin{array}{c}\text { E. coli } \\
\text { K. pneumonia }\end{array}$ & 100 & 0 \\
P. aeruginosa & 100 & $12.5 \pm 1.15, \mathrm{p}<0.05$ \\
S. aureus & 100 & $14.7 \pm 2.31, \mathrm{p}<0.05$ \\
\hline
\end{tabular}

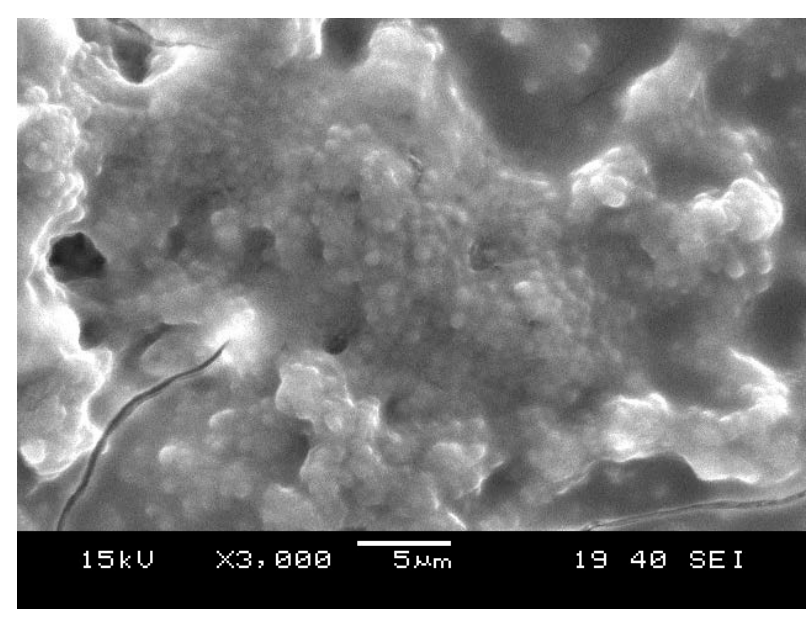

Figure 1. Electron microscopy of the control sample of Staphylococcus aureus.

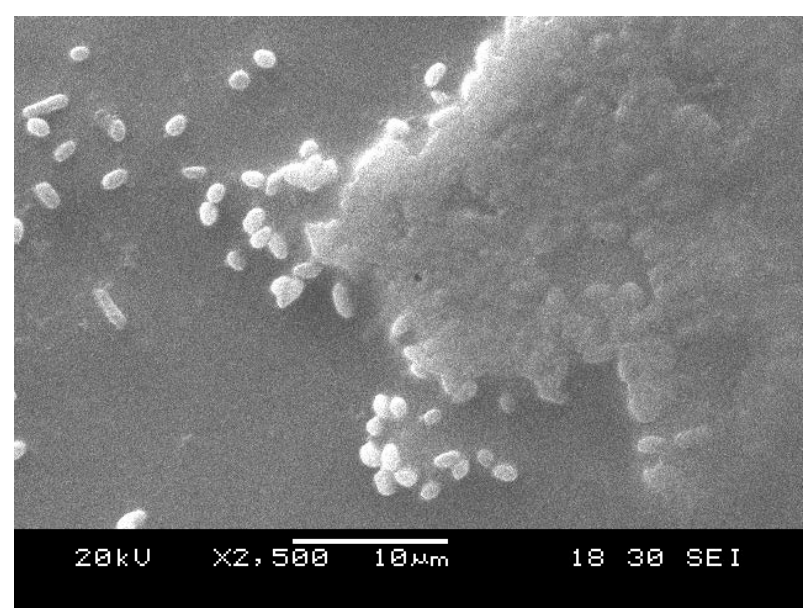

Figure 2. Electron microscopy of the control sample of Escherichia coli.

in the biofilm structure of microorganisms. Such cells are the basis for the formation of the new microorganisms' colonies.

A simulated low-intensity microwave radiation of the Sun in the frequency range $(4.00-4.30) \mathrm{GHz}$ has a strong suppressing effect on the formation of the biofilm structure of Staphylococcus aureus (Figure 3) and Escherichia coli (Figure 4). For example, in the experiment with Staphylococcus aureus we observed weakening of the process of extracellular matrix formation, its destruction (on the right side of the microscopy there is a dark area and bacterial cells that are free of matrix). The more significant effect was observed in the experiment 


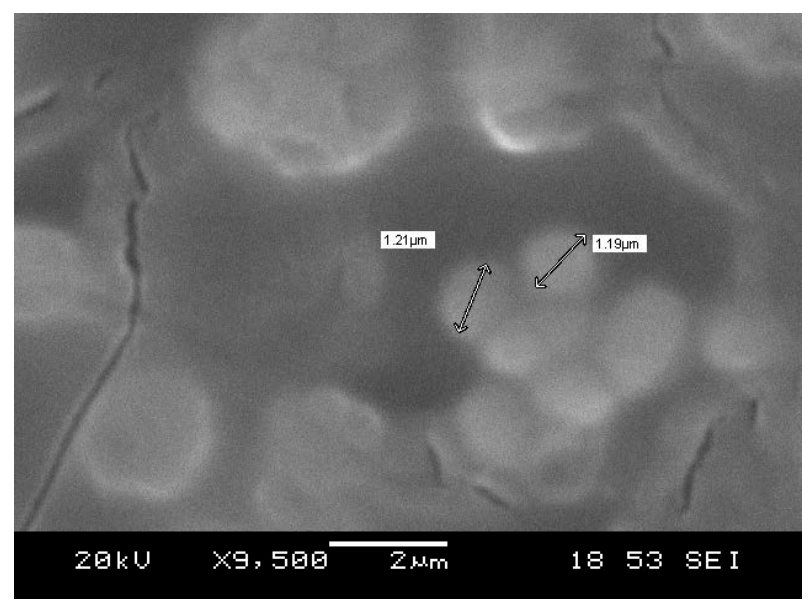

Figure 3. Electron microscopy of the experimental sample of Staphylococcus aureus.

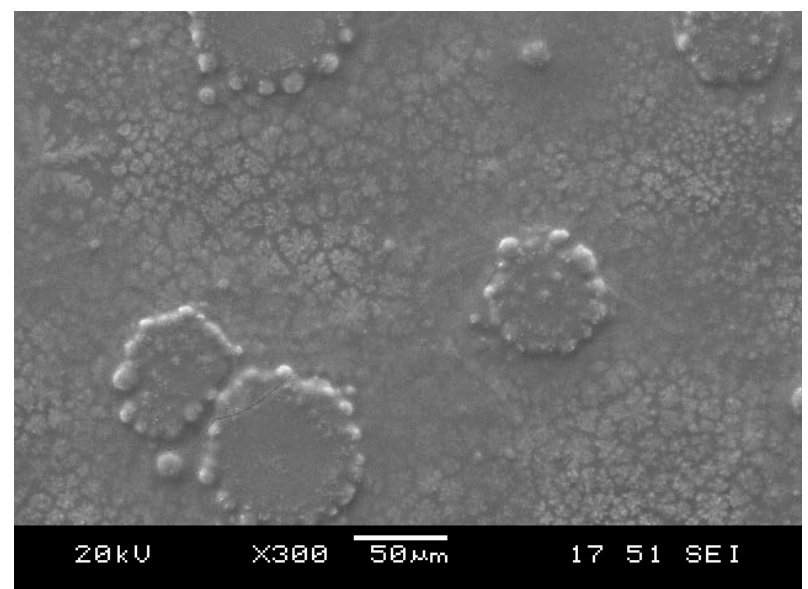

Figure 4. Electron microscopy of the experimental sample of Escherichia coli.

with Escherichia coli. The photo presented in Figure 4 shows that the electromagnetic effect on Escherichia coli led to inactivation of the biofilm formation process, on the background of the crystals of growth medium the decrease in the total number of microbial cells and the absence of extracellular matrix were visualized.

\section{Conclusions}

The results of this study confirm the hypothesis of the evolutionary nature of the leading role of the microwave radiation of the Sun in the life processes of organisms. Under the influence of this radiation, the weakening of the process of cell division and biofilm structures formation of microorganisms was observed. The mechanism of this phenomenon is not clear yet. It has been suggested that the electromagnetic radiation with the natural amplitude and time-frequency structure, due to the "radio vibratory effect", causes a separation of the bacterial microflora and thereby weakens the protective properties of microorganisms [9].

Nevertheless, we can already say that the technology of the influence on the microorganisms used in the experiment opens up the real prospects for improving the effectiveness of the treatment of the microbial diseases and complications, associated with them.

The low-intensity electromagnetic radiation used in our work differs by the UV and gamma radiation, because this technology is not intended to apoptosis microorganisms. Use of such radiation is directed mainly to counteract microbial biofilm formation. This will enhance the efficiency of immune defense mechanisms and antibiotic therapy. 


\section{References}

[1] Donlan, R.M. and Costerton, J.W. (2002) Biofilms: Survival Mechanisms of Clinically Relevant Microorganisms. Clinical Microbiology Reviews, 15, 167-193. http://dx.doi.org/10.1128/CMR.15.2.167-193.2002

[2] Costerton, J.W., Stewart, P.S. and Greenberg, E.P. (1999) Bacterial Biofilms: A Common Cause of Persistent Infections. Science, 284, 1318-1322. http://dx.doi.org/10.1126/science.284.5418.1318

[3] Romanova, Y.M. and Gunzburg, A.L. (2011) Bacterial Biofilm as a Natural Form of the Existence of Bacteria in the Environment and in the Host's Organism. Journal of Microbiology, 3, 99-109.

[4] Chebotar, I.V., Mayanskiy, A.N., Konchakova, E.D., Lazareva, A.V. and Chistyakova, V.P. (2012) Antibiotic Resistance of Biofilm Bacteria. Clinical Microbiology and Antimicrobial Chemotherapy, 14, 51-58.

[5] Römling, U. and Balsalobre, C. (2012) Biofilm Infections, Their Resilience to Therapy and Innovative Treatment Strategies. Journal of Internal Medicine, 272, 541-561. http://dx.doi.org/10.1111/joim.12004

[6] Darovskikh, S.N. (2012) Problems of the Information Management of the Organism's Homeostasis with the Help of Electromagnetic Radiation of Millimeter Range and the Main Ways of Their Solving. Biomedical Radioelectronics, 3. 3-10.

[7] Darovskih, S., Popechitelev, E., Vdovina, N. and Novikov, I. (2013) Modern Aspects of Construction of Information Microwave Therapy Devices. Natural Science, 5, 1230-1237. http://dx.doi.org/10.4236/ns.2013.512150

[8] Shishkova, Y.S., Pozdnyakova, N.L. and Molchanova, I.V. (2014) Determination of the Ability of Biofilm Formation of Microorganisms, Isolated from the Pathological Tracheobronchial Secretions. South Ural Medical Journal, 3, $29-32$.

[9] Darovskikh, S.N., Shishkova, Y.S., Popechitelev, E.P. and Vdovina, N.V. (2014) Radio Vibratory Mechanism of Interaction between the Biological Tissue of the Organisms and Electromagnetic Fields and Radiations. Bulletin of the South Ural State University, 14, 5-10. 